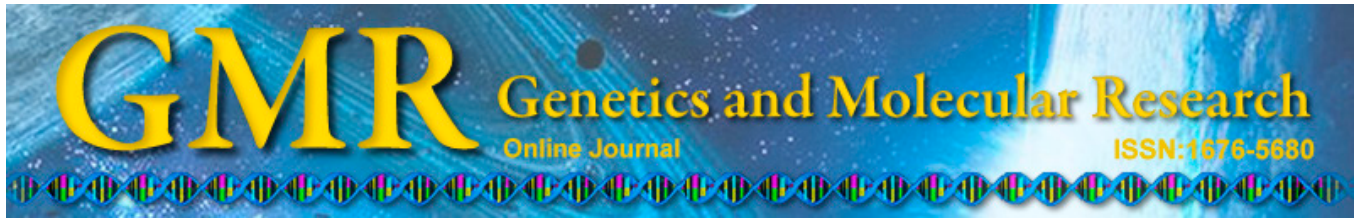

\title{
Expression and significance of plasma 3-NT and ox-LDL in patients with Alzheimer's disease
}

Z. Zhao, H. Zhou, Y. Peng, C.H. Qiu, Q.Y. Sun, F. Wang and H.N. Xie

Department of Neurology,

The Affiliated Suzhou Hospital of Nanjing Medical University, Suzhou, China

Corresponding author: Z. Zhao

E-mail: zhaozhong3355@126.com

Genet. Mol. Res. 13 (4): 8428-8435 (2014)

Received August 23, 2013

Accepted July 3, 2014

Published October 20, 2014

DOI http://dx.doi.org/10.4238/2014.October.20.19

\begin{abstract}
To examine the expression and clinical significance of plasma 3-nitrotyrosine (3-NT) and oxidized low-density lipoprotein (ox-LDL) levels in patients with Alzheimer's disease (AD), we examined $48 \mathrm{AD}$ patients and 37 healthy control subjects. The Mini-Mental State Examination, Activities of Daily Living Scale, and Hachinski Ischemic Scale were examined in all subjects. AD patients were classified using the Global Deterioration Scale. The concentrations of plasma 3-NT and ox-LDL were detected using an enzyme-linked immunosorbent assay. We found that the plasma 3-NT concentration in the AD group $(119.46 \pm 21.82 \mathrm{nM})$ was significantly higher than that in the control group $(55.09 \pm 9.63 \mathrm{nM})(\mathrm{P}<0.05)$. Spearman analysis showed that plasma 3-NT level was negatively associated with the Mini-Mental State Examination results of AD patients. Plasma ox-LDL level in the AD group $(112.25 \pm 17.81 \mu \mathrm{g} / \mathrm{L})$ was significantly higher than that in the control group $(47.46 \pm 10.04 \mu \mathrm{g} / \mathrm{L})(\mathrm{P}<0.05)$. Spearman analysis showed that plasma ox-LDL level was positively correlated with $\mathrm{AD}$ severity in AD patients. However, plasma 3-NT level in the AD group was not associated with plasma ox-LDL level. Therefore, plasma 3-NT
\end{abstract}


and ox-LDL levels in AD patients were significantly increased, which may be related to the degree of $\mathrm{AD}$ severity in $\mathrm{AD}$ patients.

Key words: Alzheimer disease; Mini-Mental State Examination; Nitrative stress; 3-Nitrotyrosine; Oxidized low-density lipoprotein

\section{INTRODUCTION}

Alzheimer' disease (AD) is a common central nervous system degenerative disease with an increasing incidence and has become the third largest serious disease influencing human life after cardiovascular diseases and cancer (Brunden et al., 2009). Studies have shown that vascular risk factors and inflammatory factors are extensively involved in AD and other neurodegenerative diseases (Fitzpatrick et al., 2004); oxidative stress and nitration stress have also been closely related to the incidence of dementia (Perry et al., 2002a). The products of oxidative stress and nitrification stress, such as nitric oxide and 3-nitrotyrosine (3-NT), are observed in $\mathrm{AD}$, and they are considered as a mediator of cellular damage and sign of apoptosis (Brenman and Bredt, 1997), which may be one of the important mechanisms for $\mathrm{AD}$. In addition, neuron degeneration and the synaptic release of cholesterol increased in $\mathrm{AD}$; lipid-containing proteins are more sensitive to oxidative stress and neurotoxicity, forming stable oxidized low-density lipoprotein (ox-LDL) (Kankaanpää et al., 2009). Serum ox-LDL has been suggested as possible candidates that can be used as biomarkers for monitoring the inflammatory process in AD (Sun et al., 2003). In this study, we examined plasma 3-NT and ox-LDL levels in AD patients and their role in the process of AD.

\section{MATERIAL AND METHODS}

\section{Subjects and methods}

Forty-eight patients were randomly selected for this study (AD group) among patients who received treatment at the Neurology Department of Suzhou Hospital affiliated with Nanjing Medical University from January 2011 to March 2011; 23 cases were males and 25 were females. Their ages ranged from 57 to 84 years, with an average age of $69.32 \pm 5.53$ years. Level of education included 10 cases of illiteracy $(20.8 \%), 16$ cases with primary school education $(33.3 \%), 14$ cases with secondary school or vocational secondary school education (29.2\%), and 8 cases of college and above education (19.7\%). Courses of disease ranged from 14 months to 10 years, with an average of $4.12+1.32$ years.

In addition, 37 healthy subjects were included as a control group (NC group), among which 19 were males (51.4\%) and 18 were females (48.6\%). Their ages ranged from 55 to 86 years, with an average age of $71.06 \pm 5.87$ years. Level of education included 8 cases of illiteracy $(20.9 \%), 11$ cases with primary school-level education $(30.0 \%), 11$ cases with secondary school or vocational secondary school education $(30.0 \%)$, and 7 cases with college and abovelevel education (19.1\%). Mini-Mental State Examination (MMSE) scores were $>24$ points. None of the control group subjects had a history of mental illness or family history of mental illness. They were healthy and had passed a recent medical examination.

The present study was performed with approval from the Ethics Committee of the Suzhou Hospital Affiliated to Nanjing Medical University and complied with the Declaration of Helsinki. Informed consent was obtained from all candidate subjects. 


\section{Inclusion criteria}

$\mathrm{AD}$ was diagnosed according to the criteria described in the Diagnostic Statistical Manual of Mental Disorders of the American Psychiatric Association, also known as the DSMIV-R diagnostic criteria (Wang and Chen, 2011). 1) Cognitive dysfunction: A. memory impairment (including near and distant memory impairment); B. cognitive impairment with at least 1 of the following situations: aphasia, apraxia, agnosia, abstract thinking, and judgment damage. 2) The above 2 cognitive dysfunctions significantly interfere with occupational and social activities or have significantly decreased over time. 3) The 2 dysfunctions do not occur during the course of delirium. 4) The 2 dysfunctions were not the result of other mental or emotional disorders. No patients had focal positive signs after complete neurological examination.

The criteria for inclusion into the NC group were as follows: 1) MMSE score was normal (>24 points). 2) The gender, age, education level, blood, and other related biological indices matched the healthy physical examination. The characteristics of the 2 groups are shown in Table 1.

\section{Neuropsychological scale measurement}

For the MMSE (Folstein et al., 1975), intellectual deficiency was selected for the 2 groups based on the educational level from illiterate, primary, secondary, and university; MMSE scores of $\leq 17,20,22$, and 23 were used as inclusion criteria for $\mathrm{AD}$ diagnosis, respectively.

The Activities of Daily Living (ADL) scale (Desai et al., 2004) comprises 20 items that are used as a supplement to assess disease severity. Score criteria were divided into 4 levels: 1 point refers to "can do it by yourself"; 2 points refers to "have some difficulties"; 3 points refers to "need some help"; 4 points refers to "cannot do it".

The Hachinski Ischemic Scale (HIS) (Moroney et al., 1997) comprises 20 items that are used to identify $\mathrm{AD}$ and vascular dementia. The higher the score, the more support for vascular dementia: $\geq 7$ points support a vascular dementia diagnosis; $\leq 4$ points support $A D$ diagnosis.

The Global Deterioration Scale (GDS) is used to classify the degree of dementia (Auer and Reisberg, 1997). GDS scores of 3-4 points refer to the "minor group", 5 points refer to the "medium group", and 6-7 points refer to the "serious group".

\section{Blood sample collection}

For blood sample collection, $2 \mathrm{~mL}$ fasting venous blood was collected; after heparin anticoagulation and $10 \mathrm{~min} 1000 \mathrm{~g}$ centrifugation, the plasma was separated from whole blood and preserved at $-80^{\circ} \mathrm{C}$.

\section{Measurement of plasma 3-NT and ox-LDL}

An enzyme-linked immunosorbent assay (ELISA) kit for 3-NT (Adlitteram Diagnostic Laboratories, San Diego, CA, USA) and an anti-human ox-LDL ELISA kit (Adlitteram Diagnostic Laboratories) were used to measure plasma 3-NT and ox-LDL concentrations.

After diluting the samples 1:5, we added $50 \mu \mathrm{L}$ sample to each well of a multi-well plate; the plate was covered and shaken gently for culturing at $37^{\circ} \mathrm{C}$ for $30 \mathrm{~min}$. Each well was filled with $0.05 \%$ TWEEN-20 PBS, washed 5 times, and patted dry. Next, $50 \mu \mathrm{L}$ enzyme 
standard reagent was added to each well except the $\mathrm{NC}$ group followed by culturing at $37^{\circ} \mathrm{C}$ for $30 \mathrm{~min}$. The plate was washed 5 times and patted dry; $50 \mu \mathrm{L}$ color-developing agent A was added to each well according to the kit instructions, and then $50 \mu \mathrm{L}$ color-developing agent $\mathrm{B}$ was added and the plate was gently shaken. The plate was incubated in the dark for the chromogenic reaction at $37^{\circ} \mathrm{C}$ for $15 \mathrm{~min}$, after which stop buffer was added to end the chromogenic reaction. The optical density value was read within $15 \mathrm{~min}$. A regression curve and regression equations for plasma 3-NT and ox-LDL were determined based on the absorbance value, which were used to calculate the concentrations of plasma 3-NT and ox-LDL.

3-NT was detected at 3-240 nM; ox-LDL was detected at 10-200 $\mu \mathrm{g} / \mathrm{L}$. Blood glucose, total cholesterol, high-density lipoprotein cholesterol, low-density lipoprotein cholesterol, 3 acyl glycerins, total protein, albumin, and other basic biochemical indicators in the AD and NC groups were detected by the clinical laboratory of our hospital (Table 1).

\section{Statistical analysis}

The SPSS 13.0 statistical software was used for statistical analysis (SPSS, Inc., Chicago, IL, USA). Measurement data are reported as means \pm SD. Count data were compared using the $\chi^{2}$ test; other clinical characteristics and indicators were compared using the Student $t$-test. Pearson correlation analysis was used in the analysis. $\mathrm{P}<0.05$ was considered to be significant.

\section{RESULTS}

\section{Characteristics of the 2 groups}

As shown in Table 1, there were no significant differences between the 2 groups regarding gender, age, education level, and other biological indices (all $\mathrm{P}>0.05$ ).

Table 1. Demographic and clinical characteristics of normal controls (NC) and Alzheimer's disease (AD) patients.

\begin{tabular}{|c|c|c|c|c|}
\hline & $\mathrm{NC}(\mathrm{N}=37)$ & $\mathrm{AD}(\mathrm{N}=48)$ & $\chi^{2}$ value & $\mathrm{P}$ value \\
\hline Age (years) & $71.06 \pm 5.87$ & $69.32 \pm 5.53$ & 1.181 & 0.243 \\
\hline Level of education (years) & $7.89 \pm 5.65$ & $6.37 \pm 6.58$ & 0.234 & 0.672 \\
\hline Smoke $[\mathrm{N}(\%)]$ & $18(50.1 \%)$ & $24(44.3 \%)$ & 0.820 & 0.347 \\
\hline Blood sugar (mg/dL) & $101.1 \pm 10.3$ & $94.5 \pm 10.2$ & 2.132 & 0.164 \\
\hline Total cholesterol (mg/dL) & $213.6 \pm 48.9$ & $204.5 \pm 20.3$ & 2.452 & 0.062 \\
\hline HDL cholesterol & $57.8 \pm 14.6$ & $45.9 \pm 10.3$ & 0.986 & 0.453 \\
\hline LDL cholesterol (mg/dL) & $136.8 \pm 16.4$ & $138.2 \pm 34.7$ & 1.221 & 0.178 \\
\hline Triglyceride $(\mathrm{mg} / \mathrm{dL})$ & $89.4 \pm 36.8$ & $125.2 \pm 45.8$ & 0.847 & 0.321 \\
\hline Total protein $(\mathrm{g} / \mathrm{dL})$ & $7.57 \pm 0.26$ & $7.50 \pm 0.43$ & 0.112 & 0.234 \\
\hline Albumin $(g / d L)$ & $3.91 \pm 0.18$ & $3.86 \pm 0.22$ & 0.213 & 0.174 \\
\hline Urea $(\mathrm{mg} / \mathrm{dL})$ & $32.8 \pm 18.2$ & $38.8 \pm 13.1$ & 0.544 & 0.139 \\
\hline Creatinine $(\mathrm{mg} / \mathrm{dL})$ & $1.10 \pm 0.17$ & $0.98 \pm 0.27$ & 0.099 & 0.082 \\
\hline $\operatorname{ALT}(\mathrm{U} / \mathrm{L})$ & $21.6 \pm 4.4$ & $20.3 \pm 4.3$ & 0.432 & 0.153 \\
\hline $\operatorname{AST}(\mathrm{U} / \mathrm{L})$ & $20.1 \pm 6.9$ & $15.9 \pm 7.2$ & 0.256 & 0.261 \\
\hline $\mathrm{LDH}(\mathrm{U} / \mathrm{L})$ & $173.5 \pm 45.6$ & $209.6 \pm 66.7$ & 2.001 & 0.421 \\
\hline Vitamin $B_{12}(\mathrm{pg} / \mathrm{mL})$ & $9.5 \pm 5.3$ & $8.6 \pm 3.9$ & 0.101 & 0.131 \\
\hline Folic acid $(\mathrm{ng} / \mathrm{mL})$ & $13.5 \pm 4.5$ & $10.2 \pm 4.3$ & 0.223 & 0.146 \\
\hline Free T4 (ng/dL) & $1.07 \pm 0.18$ & $0.99 \pm 0.23$ & 0.113 & 0.098 \\
\hline $\mathrm{TSH}(\mathrm{mIU} / \mathrm{mL})$ & $1.18 \pm 0.54$ & $1.04 \pm 0.25$ & 0.167 & 0.103 \\
\hline C-reactive protein $(\mathrm{mg} / \mathrm{dL})$ & $2.75 \pm 1.63$ & $2.59 \pm 1.97$ & 0.184 & 0.207 \\
\hline Insulin $(\mathrm{mU} / \mathrm{mL})$ & $8.91 \pm 2.94$ & $10.2 \pm 3.34$ & 0.256 & 0.386 \\
\hline $\mathrm{Hb}(\mathrm{g} / \mathrm{dL})$ & $11.2 \pm 1.9$ & $9.9 \pm 1.5$ & 0.302 & 0.235 \\
\hline
\end{tabular}

Data are reported as means \pm SD, except for smoking habit. HDL and LDL $=$ high- and low-density lipoprotein; ALT and $\mathrm{AST}=$ alanine and aspartate transaminase $\mathrm{LDH}=$ lactate dehydrogenase; $\mathrm{TSH}=$ thyroid-stimulating hormone. 


\section{MMSE, ADL, HIS, and GDS scores}

As shown in Table 2, compared with the AD group, MMSE and ADL scores for the $\mathrm{NC}$ group were statistically different (average $\mathrm{P}=0.000$ ). HIS and GDS scores were used only for AD diagnosis and to evaluate the AD severity. Based on GDS scores, patients in the AD group could be divided into 3 subgroups: minor level (11 cases), medium level (20 cases), and serious level (17 cases).

Table 2. MMSE, ADL, HIS, and GDS score in normal controls (NC) and Alzheimer's disease (AD) patients.

\begin{tabular}{lcrcc}
\hline Score & $\mathrm{NC}(\mathrm{N}=37)$ & $\mathrm{AD}(\mathrm{N}=48)$ & $\chi^{2}$ value & P value \\
\hline MMSE & $27.2 \pm 1.01$ & $14.2 \pm 5.33$ & 11.32 & $0.000^{*}$ \\
ADL & $13.25 \pm 2.30$ & $20.82 \pm 4.31$ & 10.75 & $0.000^{*}$ \\
HIS** & - & $1.32 \pm 1.52$ & - & - \\
GDS** & - & $6.35 \pm 4.62$ & - & \\
\hline Data & SD & M & & \\
\hline
\end{tabular}

Data are reported as means \pm SD. MMSE $=$ Mini-Mental State Examination; ADL $=$ Activities of Daily Living Scale; HIS = Hachinski Ischemic Scale; GDS = Global Deterioration Scale. ${ }^{*} \mathrm{P}<0.05$, NC vs AD. **Only used to evaluate $\mathrm{AD}$ patient severity and grading

\section{Plasma 3-NT and ox-LDL level}

Measurements of plasma 3-NT and ox-LDL levels in the 2 groups are shown in Table 3. The Student $t$-test showed that plasma 3-NT and ox-LDL levels in the AD group were higher than those in the NC group $(\mathrm{P}<0.05)$.

\begin{tabular}{|c|c|c|c|}
\hline & $\mathrm{AD}(\mathrm{N}=48)$ & $\mathrm{NC}(\mathrm{N}=37)$ & $P$ value \\
\hline $\begin{array}{l}\text { 3-NT (nM) } \\
\text { ox-LDL (ug/L) }\end{array}$ & $\begin{array}{l}119.46 \pm 21.82 \\
112.25 \pm 17.81\end{array}$ & $\begin{array}{l}55.09 \pm 9.63 \\
47.46 \pm 10.04\end{array}$ & $\begin{array}{l}0.000 \\
0.000\end{array}$ \\
\hline
\end{tabular}

Data are reported as means $\pm \mathrm{SD}$. 3-NT $=$ 3-nitrotyrosine; ox-LDL = oxidized low-density lipoprotein.

\section{Comparison of plasma 3-NT and ox-LDL levels in AD patients with varying severity and rank correlation analysis}

Plasma 3-NT and ox-LDL levels in the 3 subgroups of the AD and NC groups are shown in Table 4. Analysis of variance revealed that plasma 3-NT and ox-LDL levels in the 3 subgroups of the $\mathrm{AD}$ and $\mathrm{NC}$ groups were significantly different; further multiple analysis (Student-Newman-Keuls method) showed that plasma 3-NT and ox-LDL levels in the 3 subgroups of the AD group were significantly higher than those of the NC group. The analysis of plasma NT and ox-LDL levels among AD patients with different severities of disease showed that both levels increased with aggravation of the illness; the difference was significantly different $(\mathrm{P}<0.05)$. The $\mathrm{NC}$ group and patients in the $\mathrm{AD}$ group were divided into 4 levels, setting the $\mathrm{NC}$ level as 0 and minor $\mathrm{AD}$ as level 1; medium $\mathrm{AD}$ was level 2 and serious $\mathrm{AD}$ was level 3. Spearman analysis showed that plasma 3-NT and ox-LDL levels were positively correlated with $\mathrm{AD}$ severity, presented as an increased degree of dementia and increased levels of plasma 3-NT and ox-LDL (Table 5). 
Table 4. Comparisons of serum 3-NT and ox-LDL levels with different degrees of Alzheimer's disease (AD).

\begin{tabular}{lcc}
\hline Group & 3-NT $(\mathrm{nM})$ & ox-LDL $(\mu \mathrm{g} / \mathrm{L})$ \\
\hline Normal control $(\mathrm{NC} ; \mathrm{N}=37)$ & $55.09 \pm 9.63$ & $47.46 \pm 10.04$ \\
Light AD $(\mathrm{N}=11)$ & $105.98 \pm 5.43^{*}$ & $102.21 \pm 4.33$ \\
Middle AD $(\mathrm{N}=20)$ & $114.52 \pm 14.65^{*}$ & $107.64 \pm 14.93^{*}$ \\
Severe AD $(\mathrm{N}=17)$ & $133.82 \pm 27.47^{*}$ & $126.02 \pm 18.26^{*}$ \\
$F$ value & 124.365 & 186.33 \\
$\mathrm{P}$ value & 0.000 & 0.000 \\
\hline Data are reported as means $\pm \mathrm{SD} .{ }^{*} \mathrm{P}<0.05, \mathrm{NC} v$ s $\mathrm{AD} .3-\mathrm{NT}=3$-nitrotyrosine; ox-LDL = oxidized low-density \\
lipoprotein.
\end{tabular}

Table 5. Spearman correlation analysis of serum 3-NT and ox-LDL in severe Alzheimer's disease patients $(\mathrm{N}=48)$.

\begin{tabular}{lcc}
\hline & Correlation coefficient $(r)$ & P value \\
\hline NT & 0.884 & 0.000 \\
ox-LDL & 0.903 & 0.000 \\
\hline 3-NT = 3-nitrotyrosine; ox-LDL = oxidized low-density lipoprotein &
\end{tabular}

3-NT = 3-nitrotyrosine; ox-LDL = oxidized low-density lipoprotein.

\section{Linear correlation analysis of plasma 3-NT and ox-LDL levels and AD MMSE scores}

The MMSE score of the minor subgroup was $17.2 \pm 5.8$, medium subgroup was 13.3 \pm 5.6 , and serious subgroup was $11.4 \pm 6.2$. Spearman analysis showed that plasma 3 -NT and ox-LDL levels were negatively correlated with MMSE scores $(\mathrm{P}<0.05)$; with decreasing MMSE scores, plasma 3-NT and ox-LDL levels increased, as shown in Table 6.

\begin{tabular}{|c|c|c|}
\hline & Correlation coefficient $(r)$ & $\mathrm{P}$ value \\
\hline $\begin{array}{l}\text { 3-NT } \\
\text { ox-LDL }\end{array}$ & $\begin{array}{l}-0.731 \\
-0.749\end{array}$ & $\begin{array}{l}0.000 \\
0.000\end{array}$ \\
\hline
\end{tabular}

3-NT = 3-nitrotyrosine; ox-LDL = oxidized low-density lipoprotein.

\section{Linear correlation analysis of plasma 3-NT and ox-LDL levels}

Pearson analysis showed that plasma 3-NT and ox-LDL levels in the AD group were not correlated (Table 7).

Table 7. Linear correlation analysis of serum 3-NT and ox-LDL in the Alzheimer's disease group.
\begin{tabular}{llc}
\hline Item & 3-NT & ox-LDL \\
\hline 3-NT & 1 & 0.391 \\
ox-LDL & 0.391 & 1 \\
\hline
\end{tabular}

3-NT = 3-nitrotyrosine; ox-LDL = oxidized low-density lipoprotein. $\mathrm{P}>0.05$.

\section{DISCUSSION}

In the present study, we found that plasma 3-NT concentrations in AD patients were negatively correlated with MMSE score. These results indicate that plasma 3-NT 
concentration reflects the degree of cognitive impairment in AD patients. Sinem et al. (2010) found that plasma levels of nitric oxide were positively correlated with 3-NT levels. Thus, we hypothesized the following regarding the mechanism of AD. First, AD pathogenesis may be closely related with oxidative stress. When the body is influenced by infection, disease, or the environment, the oxidation level of the body exceeds antioxidant levels, and large amounts of highly active molecules such as reactive nitrogen species and reactive oxygen species can begin to accumulate. A previous study showed that oxidative stress markers for lipid peroxidation were found in the cortex and hippocampus of AD patients (Perry et al., 2002b). However, high concentrations of nitric oxide are involved in the neurotoxicity of $\beta$-amyloid (A $\beta)$, leading to AD (Lüth et al., 2001; Sultana et al., 2009). Second, previous studies have clearly observed lesions of the medial temporal lobe structures in $\mathrm{AD}$ patients, including the hippocampus, amygdala, and parahippocampal gyrus. However, the efficiency of glucose metabolism in these locations is very high, increasing the demand for oxygen. When oxidative phosphorylation occurs in the mitochondria, large amounts of oxygen free radicals and nitrogen radicals are produced (Jagust, 2006). Because various risk factors can lead to brain damage, heavier damage can lead to decreased cognitive function and increased nitric oxide and 3-NT.

It is generally considered that cholesterol content in the brain can alter amyloid precursor protein and $\mathrm{A} \beta$ expression and is involved in the morbidity of $\mathrm{AD}$ (Wahrle et al., 2002). This study revealed that the plasma ox-LDL level in AD patients was significantly higher than that in the control group $(\mathrm{P}<0.05)$; however, ox-LDL concentrations in AD patients were negatively correlated with MMSE score. This result may be related to the transfer and increase in cholesterol in the brain; in addition, LDL is more likely to be oxidized in oxidative and nitration stress environments. Dildar et al. (2010) examined 30 AD patients, 20 vascular dementia patients, and 15 patients with normal cognitive function, and found that plasma ox-LDL level in AD patients was significantly higher than that in the control group. Zafrilla et al. (2006) found that plasma ox-LDL level in AD patients showed minimal changes, while serious AD showed no clear change. Sinem et al. (2010) results also supported this finding.

In this study, we found that the plasma 3-NT level in AD patients was not correlated with ox-LDL level; however, the mechanism, which may be associated with 2 different mechanisms involved in AD onset, requires further analysis. Dildar et al. (2010) also found that plasma ox-LDL was not related to plasma 3-NT level in AD patients. In contrast, Sinem et al. (2010) found that plasma ox-LDL in AD patients was positively related to plasma 3-NT level, which may be because ox-LDL can increase oxidation activity, promote the proliferation of astrocyte cells, microglia cells, and inflammatory cytokines, which in turn exacerbate oxidative stress and stress-related nitration (Keller et al., 1999, 2000; Hsiai et al., 2007).

In summary, plasma 3-NT and ox-LDL play an important role in oxidative stress and nitration stress. As important causes of AD pathogenesis, 3-NT and ox-LDL are closely related to AD severity. Plasma 3-NT and ox-LDL are very useful in the diagnosis of AD.

\section{REFERENCES}

Auer S and Reisberg B (1997). The GDS/FAST staging system. Int. Psychogeriatr. 9 (Suppl 1): 167-171.

Brenman JE and Bredt DS (1997). Synaptic signaling by nitric oxide. Curr. Opin. Neurobiol. 7: 374-378.

Brunden KR, Trojanowski JQ and Lee VM (2009). Advances in tau-focused drug discovery for Alzheimer's disease and related tauopathies. Nat. Rev. Drug Discov. 8: 783-793.

Desai AK, Grossberg GT and Sheth DN (2004). Activities of daily living in patients with dementia: clinical relevance, 
methods of assessment and effects of treatment. CNS Drugs 18: 853-875.

Dildar K, Sinem F, Gokhan E, Orhan Y, et al. (2010). Serum nitrosative stress levels are increased in Alzheimer disease but not in vascular dementia. Alzheimer Dis. Assoc. Disord. 24: 194-197.

Fitzpatrick AL, Kuller LH, Ives DG, Lopez OL, et al. (2004). Incidence and prevalence of dementia in the Cardiovascular Health Study. J. Am. Geriatr. Soc. 52: 195-204.

Folstein MF, Folstein SE and McHugh PR (1975). "Mini-mental state". A practical method for grading the cognitive state of patients for the clinician. J. Psychiatr. Res. 12: 189-198.

Hsiai TK, Hwang J, Barr ML, Correa A, et al. (2007). Hemodynamics influences vascular peroxynitrite formation: Implication for low-density lipoprotein apo-B-100 nitration. Free Radic. Biol. Med. 42: 519-529.

Jagust W (2006). Positron emission tomography and magnetic resonance imaging in the diagnosis and prediction of dementia. Alzheimers Dement. 2: 36-42.

Kankaanpää J, Turunen SP, Moilanen V, Horkko S, et al. (2009). Cerebrospinal fluid antibodies to oxidized LDL are increased in Alzheimer's disease. Neurobiol. Dis. 33: 467-472.

Keller JN, Hanni KB, Gabbita SP, Friebe V, et al. (1999). Oxidized lipoproteins increase reactive oxygen species formation in microglia and astrocyte cell lines. Brain Res. 830: 10-15.

Keller JN, Hanni KB and Kindy MS (2000). Oxidized high-density lipoprotein induces neuron death. Exp. Neurol. 161: 621-630.

Lüth HJ, Holzer M, Gartner U, Staufenbiel M, et al. (2001). Expression of endothelial and inducible NOS-isoforms is increased in Alzheimer's disease, in APP23 transgenic mice and after experimental brain lesion in rat: evidence for an induction by amyloid pathology. Brain Res. 913: 57-67.

Moroney JT, Bagiella E, Desmond DW, Hachinski VC, et al. (1997). Meta-analysis of the Hachinski Ischemic Score in pathologically verified dementias. Neurology 49: 1096-1105.

Perry G, Taddeo MA, Nunomura A, Zhu X, et al. (2002a). Comparative biology and pathology of oxidative stress in Alzheimer and other neurodegenerative diseases: beyond damage and response. Comp. Biochem. Physiol. C. Toxicol. Pharmacol. 133: 507-513.

Perry G, Nunomura A, Cash AD, Taddeo MA, et al. (2002b). Reactive oxygen: its sources and significance in Alzheimer disease. J. Neural Transm. Suppl. 69-75.

Sinem F, Dildar K, Gokhan E, Melda B, et al. (2010). The serum protein and lipid oxidation marker levels in Alzheimer's disease and effects of cholinesterase inhibitors and antipsychotic drugs therapy. Curr. Alzheimer Res. 7: 463-469.

Sultana R, Perluigi M and Butterfield DA (2009). Oxidatively modified proteins in Alzheimer's disease (AD), mild cognitive impairment and animal models of AD: role of Abeta in pathogenesis. Acta Neuropathol. 118: 131-150.

Sun YX, Minthon L, Wallmark A, Warkentin S, et al. (2003). Inflammatory markers in matched plasma and cerebrospinal fluid from patients with Alzheimer's disease. Dement. Geriatr. Cogn. Disord. 16: 136-144.

Wahrle S, Das P, Nyborg AC, McLendon C, et al. (2002). Cholesterol-dependent gamma-secretase activity in buoyant cholesterol-rich membrane microdomains. Neurobiol. Dis. 9: 11-23.

Wang ZY and Chen G (2011). Alzheimer's disease related influencing factors analysis of cognitive damage. Zhong Guo Lin Chuang Shen Jing Ke Xue 19: 276-279.

Zafrilla P, Mulero J, Xandri JM, Santo E, et al. (2006). Oxidative stress in Alzheimer patients in different stages of the disease. Curr. Med. Chem. 13: 1075-1083. 